\title{
HACIA UNA CRIMINOLOGÍA CONTEMPORÁNEA
}

TOWARDS CONTEMPORARY CRIMINOLOGY

\author{
David Ordaz Hernández ${ }^{1}$ \\ david.ordaz@inacipe.gob.mx \\ Javier Figueroa Castellanos ${ }^{2}$ \\ javier.figueroa@inacipe.gob.mx \\ Instituto Nacional de Ciencias Penales \\ México
}

\section{SUMARIO}

- Introducción

- La criminología y su futuro

- El contexto de la criminología contemporánea

- La criminología moderna

- La criminología de la modernidad tardía

- La criminología contemporánea y la cultura

- A modo de conclusión

- Bibliografía

\section{RESUMEN}

Los autores analizan el desafío que afronta la criminología en el siglo actual, marcado por las transformaciones políticas, sociales y económicas que constituye un espacio de grandes retos teóricos que no pueden ser soslayados. Las neoconductas criminales aparecidas requieren observarse y comprenderse para redireccionar los objetivos en política criminológica. Pretenden dilucidar si los sistemas teóricos y metodológicos de las criminologías específicas explican adecuadamente la dinámica contemporánea del delito y la justicia penal con la finalidad de determinar si la criminología necesita ajustes para enfrentar una nueva realidad.

\begin{abstract}
The authors analyze the challenge faced by criminology in the present century, marked by political, social and economic transformations, which constitutes a space of great theoretical challenges that can not be ignored. New criminal misconduct needs to be observed and understood to reorient objectives in criminological politics. They aim to determine if the theoretical and methodological systems of specific criminologies adequately explain the contemporary dynamics of crime and criminal justice, in order to determine if criminology needs adjustments to face a new reality.
\end{abstract}

\section{PALABRAS CLAVE}

Criminología contemporánea, criminología moderna, modernidad tardía

\section{KEYWORDS}

Contemporary criminology, modern criminology, late modernity

\section{INTRODUCCIÓN}

La criminología contemporánea yace en un mundo de cambios acuciosos. La velocidad y complejidad de los cambios se reflejan en el carácter cambiante de la cuestión criminal y los temas recurrentes: la delincuencia juvenil,

1 Máster Internacional en Sistemas Penales Comparados, Problemas Sociales y prevención del delito, por la universidad de Barcelona, España

Técnico en Criminalística en la facultad de Derecho y Ciencias Sociales y Colegio de Criminologia de la Universidad Autónoma de Nuevo León, México

2 Maestro, psicólogo por el Instituto Nacional de Ciencias Penales, México. 
los delitos por drogas, la delincuencia en pandillas, los delitos convencionales, los delitos de cuello blanco, la violencia doméstica, los crímenes ecológicos, el expansionismo penal, el vigilantismo, los crímenes de guerra, los movimientos sociales, el abuso contra las personas de la tercera edad, los fraudes, los delitos sexuales, los delitos cibernéticos y otras formas de resistencia o desviación.

Al dejar de contemplar los datos estadísticos inmediatos de la criminalidad y mirar lo que subyace en la vida cotidiana y el control social, como los procesos de circulación de bienes y personas, la dinámica de los hogares y familias, la ecología del urbanismo, las condiciones del mercado laboral, el poder de las instituciones del Estado, se vuelve irrebatible que la criminología está comprometida en la conversión de nuestros tiempos.

La cuestión que impulsa este texto se refiere al desafío que se plantea en la criminología ante las transformaciones políticas y económicas de la actualidad que marcan la vida social a inicios del siglo XXI. El nuevo orden de las relaciones sociales y económicas, la flexibilidad de los procesos sociales, el vertiginoso cambio tecnológico y la notable multiculturalidad que confeccionan la modernidad tardía (Bauman, 2001, p. 151) plantean un escenario de complejos desafíos teóricos para la criminología que no pueden ser obviados. Seguir con los programas convencionales de investigación criminológica e ignorar los cambios del nuevo orden mundial es dar la espalda al pensamiento social contemporáneo y al análisis de la política criminológica. El contexto industrial y urbano del nacimiento de la criminología a mediados del siglo XIX la ha caracterizado por intentar ser un saber oportuno, sincrónico, de vanguardia. Los criminólogos - principalmente aquellos que recurren a la teoría social - han procurado un pensamiento congruente entre teoría y el mundo tal cual es, han basado su análisis explicando las realidades actuales ante los fenómenos de criminalidad y el desorden tanto como con los conflictos en los movimientos sociales y la dislocación entre gobiernos y población. Es así como este artículo demuestra que las transformaciones sociales del siglo Xxi bosquejan nuevas cuestiones criminales que requieren atención y comprensión por su relevancia, entendiendo las implicaciones intelectuales para la redirección de objetivos temáticos y compromisos políticos de la criminología.
¿Cómo poder llegar a un acuerdo entre criminologías ante los cambios que quejan al siglo XXI? ¿Los sistemas teóricos $\mathrm{y}$ metodológicos de las criminologías explican adecuadamente la dinámica contemporánea del delito y la justicia penal? En caso contrario, ¿qué ajustes necesita la criminología? ¿Cómo cambiar el alcance de nuestro análisis? Es así como se ponen a discusión nuevos tópicos de la criminología que respondan al mundo social contemporáneo.

\section{LA CRIMINOLOGÍA Y SU FUTURO}

Con un enfoque curioso, constructivo y alejado del nihilismo criminológico, se entiende que los criminólogos y las criminologías son categorías complejas que necesitan hoy en día de niveles superiores de comprensión y permeabilidad. Por lo tanto, se pretende problematizar aún más y lograr una clarificación conceptual. En este momento es necesario recalcar que la Academia tiene poco que ganar cuando trata de sustituir el término criminología por conceptos artificiales como el de justicia actuarial (Hannah-Moffat, 2013, p. 30). Asimismo, el proyecto de incorporación de la criminología al análisis de la globalización con frecuencia acarrea crisis teóricas (Barak, 2009; Morrison, Zaffaroni \& Bernal, 2012, p. $6)$, dichas crisis - por las que ha pasado con bastante frecuencia - no puede desalentar a los criminólogos en nuevos esfuerzos hacia una criminología contemporánea. A mi parecer la angustiosa búsqueda de la identidad científica de la criminología trajo limitaciones en las incursiones de los criminólogos a otros lugares, dicho posicionamiento ahora es impensable o erróneo al reducir los esfuerzos de los estudios criminológicos en una sola dirección.

La idea de una criminología como ciencia autónoma y resistente por el uso del método científico obedece a etapas anteriores de su desarrollo histórico (Elbert, 2012, p. 4), y estas condiciones particulares de desarrollo criminológico son las que debemos interrumpir. Esto implica que los estudiantes de las criminologías y el control del delito, como sostiene Pitch (1995), tendrán que aprender a pensar más allá de los umbrales de la criminología como se constituye en la actualidad su cuestión criminal. Sabiendo que la criminología está destinada a la movilidad epistemológica casi suicida (Pavarini, 1994), debe ser una disciplina que continuamente se reedifique para 
comprender los niveles académicos - teóricos y metodológicos - del mundo social y jurídico en los que aspira a intervenir.

Tales afirmaciones parecen innecesarias ante la escalada y arraigo que mantiene la criminología en la vida académica, en ese sentido este saber goza de una gran salud en términos cuantitativos. Hablando de actividades relativas a la criminología se celebran en México cerca de cinco congresos al año, hasta febrero del 2015 la Subsecretaría de Educación Superior a través de su registro de Reconocimiento de Validez Oficial de Estudios Superiores Federales y Estatales del país ha inscrito 163 planes de estudio en diferentes niveles educativos en criminología, los estudiantes con cédula son más que en otros tiempos, los departamentos de investigación de las universidades contemplan más que nunca la criminología, existe un gran número de consultoras privadas en la materia, las revistas especializadas y las publicaciones académicas son prolíferas en el análisis de la criminalidad, el abordaje de la cuestión criminal en volumen nunca había sido tan vigoroso. Sin embargo, la salud y la arrogancia que escolta la criminología como disciplina político-académica, se desvanece en la medida que entra al terreno de la discusión pública sobre sus aportes y toma de decisiones en la política pública del país.

Es un hecho que para las sociedades occidentales, la seguridad, la criminalidad y su castigo es tan importante, ininteligible y polémico que ningún saber científico puede decretar la forma de comprenderlo o atenderlo. El crimen y el castigo tienen una función tan importante y sistémica en las sociedades actuales que están estrechamente vinculadas a la vida cotidiana (Garland \& Sozzo, 2005, pp. 236-237), la percepción y la emoción de los riesgos, el vívido temor de un imaginario colectivo basado en la incertidumbre (Bauman, 2001; 2011, p. 75), este conglomerado desaparece con facilidad de cualquier método analítico, pero sin embargo la criminología podría lograr su contención. Por eso, la criminología debe de desistir en sus intentos de monopolizar el discurso criminológico y debe de dejar de reclamar la exclusividad del análisis de la cuestión criminal.

De las ideas anteriores podemos desglosar que algunos modelos intelectuales $y$ supuestos institucionales —principalmente los provenientes del sistema penitenciarioque sirvieron a las generaciones anteriores de criminólogos no son del todo apropiadas para el presente. Más aún, los cambios sociales de los últimos años ya se han llevado a una reflexión y un replanteamiento en diversos saberes, cuando la criminología académica se consolidaba con dificultad como una especialidad de perspectivas plurales.

Por consiguiente, algunos de nuestros hábitos académicos criminológicos tienen que ser evaluados. Por citar un ejemplo, el cambio de las formas de legitimación del sistema penal y los acuerdos sociales que hay al respecto han afectado las formas en que los criminólogos piensan sobre las cuestiones de regulación de la autoridad. La ambigua distinción y el deterioro entre lo público y lo privado del control de los conflictos en las urbes (Sennett, 2011, pp. 361-379), hasta el desplazamiento de la justicia penal estatal de la producción de seguridad y control del crimen por los sistemas de vigilancia privados, que suponen el control de lo que pasará (Bauman \& Lyon, 2013), han tenido tal impacto que la criminología se ocupa del análisis de la regulación y el control de la criminalidad.

Así, los criminólogos procedentes de diversas vertientes o estudios como la policía, las cárceles, las víctimas, los sistemas de justicia o la prevención deliberan más allá de la relación Estado-delincuente. El resultado de una criminología contemporánea no es estructurar nuevas formas de concebir la distribución del orden social con sus disyuntivas normativas y éticas, sino de repensar el proceso de construcción de la cuestión criminal para mejorar las condiciones de conceptualizar las características fundamentales del control y orden social en el nuevo milenio.

Considero que otra oportunidad de este mundo académico cambiante es la soltura que muestran los jóvenes hacia un intercambio intelectual entre investigación empírica y los intereses renovados hacia la comprensión de la teoría social. El trabajo es reunir el pensamiento que se reconoce como fragmentado para llevar a la criminología hacia una integración científica que procure el cambio social (Elbert, 2012, pp. 180182). Esta actividad requiere de responsabilidad académica, el criminólogo se encuentra obligado a considerar las condiciones contemporáneas 
de la sociedad y a ser reflexivo sobre la función política de la criminología para elegir dignamente la cuestión criminal; en este punto considero necesario que se desarrolle una revalorización de los textos clásicos que fundaron los movimientos epistemológicos de la criminología, pues permiten poner en relieve los problemas actuales de la criminología ante la organización social, la vida cotidiana de la sociedad mexicana y las distintas formas de gobierno.

\section{EL CONTEXTO DE LA CRIMINOLOGÍA CONTEMPORÁNEA}

El surgimiento de la criminología teórica de los años cuarenta a la actualidad a dado aportes satisfactorios y limitaciones, por el lado de los aportes encontramos amplios estudios empíricos y validaciones teóricas, pero con severas críticas a su utilidad en el mundo real. Una criminología contemporánea sugiere estrategias e iniciativas para prevenir y disminuir el crimen en la medida que se analiza al sistema de procuración de justicia. Uno de los pioneros de la criminología del siglo xx hacia el análisis contemporáneo de las sociedades es Edwin Sutherland (1966, p. 1), quien ha demostrado haber construido una definición duradera:

La criminología es el cuerpo de conocimientos relacionados a la criminalidad como fenómeno social. Se incluyen dentro de su ámbito de aplicación los procesos de elaboración de leyes, de incumplimiento de las leyes, y de reacción hacia el incumplimiento de las leyes. Estos procesos son tres aspectos de una secuencia un tanto unificada de interacciones.

Gran parte de la construcción de la teoría criminológica moderna — por la búsqueda de sí misma - se logra entre la década de 1950 y 1970 y su desarrollo permitió que los criminólogos advirtieran que el análisis de la sociedad y el crimen no solo eran de carácter explicativo sino que influían en los agentes del control social y el criminal (Matthews, 2014). El punto cúspide del uso académico para combatir la delincuencia se encuentra a finales de la década de 1960 cuando los movimientos sociales y las crisis económicas ponían en controversia el uso de la fuerza y de la criminalización.

Para Garland (2005, p. 83) los fundamentos de la criminología contemporánea se encuentran en Gran Bretaña por la combinación de una criminología administrativa y los registros institucionales. Los autores del siglo $\mathrm{xx}$ siempre mantuvieron la necesidad de llevar la teoría de la criminalidad y el castigo al mundo real del control de la delincuencia, dar una salida al conocimiento criminológico.

No hay mejor manera de abordar la criminología actual que hablar sobre su pasado. Una de las primeras producciones criminológicas que analizan el porvenir de la sociedad contemporánea y el delito es la obra de Radzinowicz (2002), quien afirma la discordancia entre la teoría criminológica y su aplicabilidad:

Lo que me parece profundamente preocupante es la diferencia entre «criminología» y «política criminal», entre el estudio del crimen y castigo, y los modos actuales de control de la delincuencia. Esta brecha, para decirlo en términos más generales, entre la teoría y la práctica siempre había existido, pero se ha vuelto cada vez más arraigada como el trascurrir de los años, y no hay indicios de que se reduzca en el futuro previsible. Esto, sin embargo, ha sucedido no debido a algunas fallas fatales en los hallazgos de pensamiento y de investigación criminológica, sino simplemente porque las normas en la administración de justicia penal en muchas partes del mundo son desesperadamente bajas y últimamente han ido incluso más lejos, cuesta abajo (ibid., p. 468)

Radzinowicz no será la última persona en dar cuenta del desarrollo del populismo penal, pero pone al descubierto que la criminología juega un papel importante en la política criminológica. Por eso, para el criminólogo actual debe ser llamativa la discordancia entre los resultados de la investigación criminológica y las políticas criminológicas. Es así como los gobiernos actuales han normalizado a través de la retórica del uso de la ley y el orden utilizando medidas penales más extensas, mayor utilización de la prisión lo que demuestra un claro distanciamiento entre los resultados de los expertos en criminología y las políticas del castigo. De forma específica, Ferrajoli (2013, pp. 75-76) refiere las dos razones del fracaso del populismo punitivo.

En primer lugar, porque su efecto principal es el debilitamiento del tejido social, que forma el primer presupuesto no solo de la democracia, 
sino también de la seguridad. El miedo, en efecto, rompe los lazos sociales, alimenta tensiones y laceraciones, genera fanatismos, xenofobias y sectorizaciones, genera desconfianzas, sospechas, odios y rencores. Envenena en poco tiempo a la sociedad haciéndola retroceder al estado salvaje y a la ausencia de civilidad. Y en esta regresión, como es obvio, representa el principal terreno de la cultura de la criminalidad y de la violencia, además de la amenaza más insidiosa para la democracia.

En segundo lugar, estas políticas demagógicas desvían la atención de la opinión pública y el compromiso de los recursos de los aparatos investigativos judiciales en los procesos contra la criminalidad organizada y contra sus convivencias con los poderes políticos y económicos. Las mismas sirven, de hecho, a orientar la acción de tales aparatos hacia la pequeña criminalidad que depende del gran crimen organizado.

En la persecución del distinto se plantean las políticas criminológicas del populismo penal, engendrando el terror y la suspicacia del otro, del ajeno a la civilización.

Hacer noción a la evolución del análisis del populismo penal permite contrastar a la luz histórica las distintas generaciones de la criminología académica moderna, pues muestra la solidez y tacto del conocimiento de la cuestión criminal para tener un mayor impacto en la política pública de los distintos gobiernos. En ese sentido cabe reflexionar sobre la producción criminológica de América Latina puesto que es bastante razonable reconocer que ocurre algo contrario a lo anteriormente señalado, esto quiere decir que nuestra región padece de la sensación de ir en un sentido inverso al desarrollo de los sistemas de conocimiento criminológico, sabiendo que nuestros conocimientos han madurado y nuestras sociedades se han vuelto más civilizadas y modernas, pareciera que repentinamente sufrimos un retroceso.

La evolución de la criminología en América Latina se encuentra bloqueada. Esa inversión entre el éxito del papel de la criminología (ya antes mencionado) y su rol en la vida pública es un tema a desarrollar en la criminología actual. Esta cuestión proporciona diversos puntos de análisis en la que es necesario utilizar los recursos históricos para reflexionar sobre los temas de nuestro tiempo. De esa forma, es importante explicar que los conocimientos criminológicos con una mayor aceptación en la Academia tienen una situación geográfica específica, o mejor dicho una preponderante hegemonía política-económica, que por razones de poder y legitimidad incluyen $\mathrm{y}$ excluyen estudios criminológicos.

Estos ejercicios de poder siempre se encuentran tentados a generalizar y crear vínculos aparentes entre las conclusiones de los estudios y el orden social de las civilizaciones bajo la narrativa de la ilustración progresista (Morrison, Zaffaroni \& Bernal, 2012, pp. 54-55).

En un sentido general la criminología organiza el saber sobre el crimen, los criminales y las distintas formas del control del delito. De forma científica y elaborada, los criminólogos analizan el trabajo de las instituciones penales y la cultura popular del crimen así como el imaginario social que existe de la criminalidad. Para ejemplificar lo dicho se puede decir que la criminología se inscribe en cuatro perímetros sociales: a) Los corporativos de la Academia y sus discursos (Zaffaroni, 2005, p. 23). b) La esfera del control del delito y el orden social a través del sistema penal (Garland \& Sozzo, 2005, p. 313). c) El ámbito cultural que incluye las formas como las fuerzas culturales se enlazan con la práctica de los sistemas penales en la lucha contra el crimen. Esto es permitir que la criminología salte del estrecho margen del delito y la justicia penal para analizar las muestras simbólicas de la transgresión y el control, los sentimientos y emociones que surgen de los hechos delictivos, así como el análisis de las campañas políticas diseñadas para definir el crimen y sus efectos o castigos (Ferrell, Hayward \& Young, 2008, p. 2). d) Por último, la cuestión más letal que ha sido olvidada por la criminología, el ámbito de la guerra; analizado y criticado bajo las condiciones morales del nacimiento del Estado moderno (Ruggiero, South \& Taylor, 1998, p. 499).

Estas propuestas de matrices criminológicas se encuentran vagamente identificadas por el criminólogo, pero desde mi opinión son la clave para que la disciplina siga siendo un parámetro en las decisiones gubernamentales e influencia en la sociedad.

El desarrollo del pensamiento criminológico contemporáneo refleja el crecimiento de las 
ciencias sociales bajo la teoría crítica. Tanto de las investigaciones como del dominio de la teoría sociológica, de la psicología social y de los estudios culturales; se manifiestan principalmente corrientes intelectuales en criminología como el marxismo, el feminismo y el posmodernismo. Sin embargo, se considera necesario como anuncia Elbert (2004) que la criminología latinoamericana subraye la reconstrucción social, la redistribución y la inclusión.

Pero la criminología puede pensarse en múltiples contextos. Su historia muestra la capacidad de analizar la cuestión criminal desde los controles gubernamentales del crimen, el ámbito institucional como elemento de gobierno y en ocasiones es fuente de asesorías estratégicas para el control del crimen y del castigo.

\section{LA CRIMINOLOGÍA MODERNA}

Es importante señalar que la criminología moderna no hace referencia a lo coetáneo, más bien la criminología moderna reseña el conjunto de problematizaciones y conceptos referentes al razonamiento de la sociedad de finales del siglo xIx y comienzos del siglo $x x$, época que fue trazada por los saberes de la psicología clínica, la antropología criminal, los amplios estudios estadísticos del crimen y la fuerte idea del progreso a través de la disciplina (Garland, 2005, p. 71). Dicho marco histórico fraguó los ejes de las instituciones penales en el Estado benefactor durante setenta años del siglo xx, cuya lógica principal era que la ciudadanía en el ámbito social mantenía un sistema de beneficios basado en seguros (Walklate, 2007, p. 107). La criminología moderna ya no se encuentra a la vanguardia del reconocimiento de la cuestión criminal, pero su discurso de supremacía permitió la configuración del sistema penal de setenta años.

La criminología que pertenece a los tiempos de la modernidad tenía un gran apego por la razón instrumental, su compromiso social se encontraba bajo los diseños de la ingeniería social. Mientras que el castigo desde una visión retributiva era visto como irracional y contraproducente para la sociedad (Garland, 1999). La función de castigar se conducía bajo medidas individualizadas, correctivas, bajo el análisis de problemas particulares; dicha postura inicia con el programa de Marburgo como lo señala Ferrajoli (1995).
La criminología moderna conceptualizaba la delincuencia como un problema social que se expresa en actos individuales, eran los síntomas de la criminalidad. Signos de sujetos mal adaptados o que carecieron de una sana socialización. En estos momentos de modernidad, la criminología experimentó un fuerte acercamiento con la psicología, se centró en causas profundas, experiencias lejanas, infancia y conflictos psicológicos. Así se produjo una cuestión criminal con un objeto de estudio propio a lo que Zaffaroni (2011) llama paradigma etiológico. El problema era el delincuente y este sujeto era un inadaptado cuyo tratamiento correccional era la solución.

La criminología siguió el curso de la modernidad y las formas de investigación cambiaron a lo largo del siglo; en un principio, como se ha mencionado, fue la medicina y la psicología de la anormalidad que elaboraron el objeto de estudio, mientras que más adelante bajo una nueva explicación etiológica se acercó la psicología social y la sociología (Zaffaroni, 2011, p. 219). El proceso de explicación a través de la privación social (privados de una educación adecuada, o la socialización familiar, o las oportunidades de trabajo, o el tratamiento adecuado para sus problemas sociales y psicológicos) evolucionó en la privación relativa (la experiencia de sentir carencia por algo que uno cree tener), en todo caso es visible que la criminología se mantuvo al margen del evento delictivo y de las oportunidades criminales.

La criminología moderna fuera de la política y neutral ante los movimientos sociales pudo combinar su experiencia científica para mimetizarse en las reformas liberales. Bajo una expresión, la criminología supo navegar en un centroderecha política que permitió la aceptación de los diversos posicionamientos de la izquierda no radical y derecha. Se puede decir que la posición conservadora de la criminología moderna fue representada en los discursos políticos de ciertos sabios morales como jueces y magistrados que mantenían una explicación de la delincuencia originada por una moral incipiente (Morrison, 1995, p. 110).

\section{LA CRIMINOLOGÍA DE LA MODERNIDAD TARDÍA}

Como se habrá dado cuenta el lector, el mundo que habita la criminología hoy se encuentra 
lejos de las descripciones previamente mencionadas, no es el mismo mundo que vio nacer la criminología moderna ni persisten fuertemente las aspiraciones de orden y progreso. Los profundos cambios sociales, políticos y económicos de inicios de la década de los setenta deshicieron las certezas de las teorías sociales y el progreso. De forma sintética, se puede decir que el mundo es más complejo y problemático. Tan problemático que los teóricos no pueden ponerse de acuerdo con la caracterización de las formas de vida y el nuevo orden mundial.

Por lo tanto, existe una serie de conceptualizaciones en diversos autores como: posmodernidad de Jean Baudrillard, modernidad tardia de Anthony Giddens, modernidad líquida de Zygmunt Bauman, sociedad del riesgo de Urlich Beck, sociedad posindustrial de Daniel Bell y Alain Touraine, capitalismo de la posorganización de Scott Lash, entre otras descripciones. Todos estos términos complejizan la realidad, discuten sobre la periodicidad y la identificación de las transformaciones del mundo. Por eso, los criminólogos tienen mucho que hacer en la construcción de la cuestión criminal bajo estas conceptualizaciones de los tiempos actuales, y muchos han iniciado una respuesta digna como respuesta intelectual a los recalcitrantes criminólogos modernistas.

Varios estudios criminológicos relativamente recientes dan cuenta de la complejidad de la nueva era mundial (Morrison, Zaffaroni \& Bernal, 2012; Freilich \& LaFree, 2014; DeKeseredy, 2011; Ahmed, 2014, pp. 5455). Estos trabajos muestran o advierten los giros dramáticos que ha sufrido el mundo, la complejidad y peso que recae en la disciplina criminológica. Hay que tener en cuenta que los principios más afectados de la criminología moderna son su racionalidad instrumental y el tratamiento criminológico.

Para comprender esto, la criminología crítica atiende a la modernidad tardía como el surgimiento cúspide de un cambio radical en el último tercio del siglo xx. Las criminologías que advierten este cambio son atentas a la construcción social de la realidad, aceptan una confusión epistemológica por el uso de la razón científica, reconocen un pluralismo y la naturaleza controvertida de las normas, así como examinan la vida cotidiana para comprender mejor la criminalidad. Los principios criminológicos que dieron nacimiento a la criminología crítica de la década de 1970 no podrían tener mayor validez como hoy en día.

Por lo tanto, los resultados de la criminología crítica deben ser encaminados a la oposición de los efectos neoliberales del sistema penal.

La necesidad de desarrollar una criminología a pesar de la caída de los grandes relatos legitimadores (Lyotard, 1989, p. 31) o del nihilismo posmoderno es remontar esfuerzos sobre el análisis de la transformación social en los sistemas penales cambiantes y el controvertido uso de la fuerza a través de los aparatos de justicia, son temas de discusión inminentes puesto que han cambiado las formas de pensar y actuar ante el delito. Independientemente de las conceptualizaciones señaladas, la modernidad tardía permite informar sobre una reconfiguración social, económica y cultural hecha por la congregación de diversos acontecimientos interrelacionados (Garland, 1997):

1. La transformación de la producción y el intercambio capitalista

2. Los cambios de secularización de la familia y los hogares

3. Cambios en la ecología social y la demografía

4. El impacto de los medios masivos de comunicación y

\section{La democratización de la vida cultural}

La transformación de la civilización se relaciona con la reorganización de las clases sociales, se logró cuando grupos poderosos obligaron a recesiones durante 1970 y 1980 a través de la imposición de una economía agresiva de libre mercado, que tenía entre sus fines la desaparición del Estado benefactor y la imposición de una cultura conservadora. Es innegable que la modernidad tardía trajo nuevas formas de consumo (Bauman, Rosenberg \& Arrambide, 2007), libertades individuales y elecciones (Lipovetsky, 2010), pero a su vez estos cambios estructurales modificaron el sentido de seguridad y delincuencia.

Una de las contradicciones esenciales que considero para el análisis criminológico de 
estos tiempos es reconocer las supuestas libertades de la vida cotidiana versus las supuestas restricciones estatales para la gobernanza, puesto que los problemas de control de la criminalidad posiblemente provengan de la permisividad amoral de las instituciones sociales.

Así, el panorama de la criminología actual muestra altos niveles de delincuencia y un sinfín de políticas criminológicas paliativas o contradictorias. Además, miles de jóvenes han crecido en periodos de hiperviolencia y grupos sociales consideran la violencia como una condición normal de la vida diaria. El temor al delito permea la existencia de la sociedad y la explotación afectiva a través de los medios de comunicación permite la politización de las políticas de securitización (Heitmeyer, 2011, p. 168). Por último, podemos decir que el dominio gubernamental de la justicia penal está a merced de un mercado creciente de seguridad privada.

Sin duda, la modernidad tardía ha configurado en las mujeres y hombres una consciencia psicológica del crimen, que obliga a una adaptación de rutinas defensivas, mientras que la exposición a altos niveles de miedo e incertidumbre resulta en un continuo de frustraciones. Estas adaptaciones al temor del delito configuran nuevas formas de defensa, o evitación del delito que suelen ser respuestas hostiles. Parte de la población se vuelve más tolerante a la violencia percibida, pero intolerante a la comprensión del delincuente. Y esta postura exige generar políticas para el aumento del control de los delincuentes y sus castigos sin importar la posible pérdida o invasión de los derechos humanos. De forma casi natural, criminologías del control del delito emergen a esas voces, criminologías que refuerzan la incertidumbre basadas en la elección racional, en la prevención situacional para concluir fríamente en la incapacitación o exclusión del delincuente.

\section{LA CRIMINOLOGÍA CONTEMPORÁNEA Y LA CULTURA}

Concebir la cuestión criminal sin la cultura es caminar a ciegas. La complejidad cultural y las múltiples bifurcaciones de las imágenes de la violencia y el miedo saturan la vida social de las sociedades. Tratar de monopolizar o sistematizar las formas de analizar esas cuestiones es casi imposible, por eso la academia criminológica congruente ha sido flexible con la cuestión criminal y rompe sus barreras para discutir a profundidad la criminalidad. El problema central de la investigación a través de los métodos culturales tiene una serie de aristas como el feminismo, urbanismo, ecología (criminología verde), arquitectura, cine, política, análisis de riesgos, entre otras que permiten comprender la cultura política de la sociedad.

Bajo el contexto laboral de la modernidad tardía, el criminólogo tiene opciones estratégicas en el mercado laboral, puede ser así mismo una especie de obrero, especialista en debates del populismo punitivo o como fuente de datos para debates más amplios como la defensa de la criminología mediática (Zaffaroni \& Rep., 2011). Este papel con frecuencia se asigna a los ideólogos jerarcas del saber o académicos cómodos. O un segundo criminólogo puede tener un fuerte compromiso con la discusión actual centrando su discurso en la cuestión social y cultural de la delincuencia.

El conocimiento criminológico actual requiere la comprensión y el entendimiento cercano a los estudios críticos con nuevas respuestas institucionales, puesto que muchas instituciones se resisten al esclarecimiento de la cuestión criminal. A pesar de las adversidades ideológicas y políticas que se presentan al criminólogo consciente, los circuitos de comunicación académica nunca habían sido tan extensos y contiguos a la organización cultural y social de las comunidades lo que permite intervenciones de mayor precisión y claridad en la expresión de los conocimientos académicos.

Estas cuatro décadas posteriores a la aparición de la modernidad tardía significan repensar la criminología en contextos diferentes. Durante mucho tiempo la criminología sufrió de prisionización ya que se localizaba bajo las paredes de las instituciones de la justicia penal del Estado, a medida que avanza la criminología se crean nuevos lugares epistemológicos cuyo marco institucional se vuelve objeto de estudio. Por ello, es claro que el resurgimiento de la policía privada y la comercialización de la seguridad permiten nuevas formas de conocimiento acerca de la delincuencia y la victimización que tienen que ser examinadas. Los procesos secundarios de victimización, frecuentes en la modernidad tardía, obligan a repensar los temas en criminología. 
Bajo su responsabilidad académica, la criminología ha generado las oportunidades de participar en los debates públicos sobre la cuestión criminal que aquejan nuestros tiempos. Aquel criminólogo que intente entrar al campo de batalla deberá comprender los términos en los que se discuten, asistir a ellos bajo una crítica ingenua será legitimar las nuevas formas de criminalización. Por ello, los criminólogos deben desarrollar una autoconsciencia sobre los principios que rigen estos tiempos y conocer a profundidad la situación social, pues la cultura política definirá el destino de la criminología. Es un camino que está por decidirse.

\section{A MODO DE CONCLUSIÓN}

Sería crédulo esperar que los conocimientos criminológicos futuros fueran uniformes. Se espera entonces que los criminólogos que estudian bajo la mirada de la modernidad tardía, entreguen estudios criminológicos de diversas texturas y una variada complejidad, pues los contextos contemporáneos de la violencia no pueden tener un fácil consenso o una agenda compartida. Es admirable reconocer que un grupo de estudiantes ha dejado los campos tradicionales de estudio, la sociología del castigo y las empresas criminales se vuelven temas de interés. Solo queda invertir los intereses de la criminología mediática para adherirse a nuevos horizontes criminológicos.

Del mismo modo, es digno reconocer que los trabajadores y académicos que habitan el sistema penal se saben presa de obsoletos términos tradicionales mientras esperan innovaciones tecnológicas, políticas u organizacionales que impacten las formas del control del delito.

Tal vez para una criminología contemporánea se requieran recursos intelectuales con compromiso en la teoría social, en ese sentido continuar con el viaje de la reflexión teórica y la investigación empírica entre criminología y teoría social hacia la explicación de la cuestión criminal.

\section{BIBLIOGRAFÍA}

Adams, M. (2009). A Delinquent Discipline: The Rise and Fall of Criminology. Acad. Quest., 22 (4), pp. 491-503. DOI: 10.1007/s12129009-9133-7
Ahmed, S. (2014). Constitutive Criminology and the 'War on Terror'. Critical Criminology, 22 (3), pp. 357-371. DOI: 10.1007/s10612014-9235-6

Barak, G. (2009). Criminology (p. 6). Lanham, Md.: Rowman \& Littlefield Publishers.

Bauman. Z. (2011). La globalización. Consecuencias humanas. México: Fondo de Cultura Económica.

Bauman, Z. (2011). Daños colaterales. Madrid: Fondo de Cultura Económica.

Bauman, Z., \& Lyon, D. (2013). Vigilancia líquida. Barcelona: Paidós.

Bauman, Z., Rosenberg, M., \& Arrambide, J. (2007). Vida de consumo. México: Fondo de Cultura Económica.

Carrington, K., \& Hogg, R. (2002). Critical criminology. Cullompton, Devon, UK: Willan Pub.

DeKeseredy, W. (2011). Contemporary critical criminology. New York: Routledge.

Elbert, C. (2012). Criminología, ciencia y cambio social (pp. 180-182). Buenos Aires, Argentina: Eudeba.

Elbert, C. (2004). Rebuilding Utopia? Critical criminology and the difficult road of reconstruction in Latin America. Crime, Law And Social Change, 41 (4), pp. 385-395. DOI: $10.1023 / \mathrm{b}$ :cris.0000025768.98914.d5

Elbert, C. (2012). Criminología, ciencia y cambio social. Buenos Aires, Argentina: Eudeba,pp. 180-182.

Ferrajoli, L., Bobbio, N., \& Andrés Ibáñez, P. (1995). Derecho y razón. Madrid: Trotta.

Ferrell, J., Hayward, K., \& Young, J. (2008). Cultural criminology (p. 2). Los Ángeles: SAGE.

Freilich, J., \& LaFree, G. (2014). Criminology Theory and Terrorism: Introduction to the Special Issue. Terrorism And Political Violence, 27 (1), pp. 1-8. DOI: $10.1080 / 09546553.2014 .959405$

Gregg Barak. Criminology. Lanham, Md.: Rowman \& Littlefield Publishers, 2009, p. 6. 
Garaland, D. (1999). Castigo y sociedad moderna. Madrid: Siglo XXI Editores.

Garlan, D. (1997). 'Governmentality' and the Problem of Crime: Foucault, Criminology, Sociology. Theoretical Criminology, 1 (2), pp. 173-214. DOI: $10.1177 / 1362480697001002002$

Garland, D., \& Sozzo, M. (2005). La cultura del control. Barcelona: Gedisa.

Garland, D., Iturralde, M., \& Garland, D. (2007). Crimen y castigo en la modernidad tardía. Bogotá: Siglo del Hombre.

Giddens, A., Beriain, J., \& Sánchez Capdequí, C. (1996). Las consecuencias perversas de la modernidad. [Barcelona]: Anthropos.

Hannah-Moffat, K. (2013). Actuarial Sentencing: An «Unsettled» Proposition. Justice Quarterly, 30 (2), pp. 270-296. DOI: $10.1080 / 07418825.2012 .682603$

Harcourt, B. (2003). The Shaping of Chance: Actuarial Models and Criminal Profiling at the Turn of the Twenty-First Century. The University Of Chicago Law Review, 70 (1), p. 105. DOI: $10.2307 / 1600548$

Heitmeyer, W. (2011). Control of violence (p. 168). New York: Springer.

Lipovetsky, G. (2010). La era del vacío. Barcelona: Anagrama.

Luigi F., Bobbio N., Ibáñez, A., (1995), Derecho y razón. Madrid: Trotta.

Luigi F., Bobbio N., Ibáñez, A., (2013), Derecho y razón. Madrid: Trotta.

Lyotard, J. (1989). La condición postmoderna. Madrid: Cátedra.

Matthews, R. (2014). Realist criminology. England: Palgrave Macmillan.
Morrison, W. (1995). Theoretical criminology (p. 110). London: Cavendish Pub.

Morrison, W., Zaffaroni, E., \& Bernal, C. (2012). Criminología, civilización y nuevo orden mundial. Barcelona: Anthropos.

Pavarini, M. (1994). Nuevos modelos explicativos en criminología. La visión de la criminología crítica. Iter Criminis. Revista de Derecho y Ciencias Penales, 3, pp. 159-181.

Pitch, T. (1995). Limited responsibilities. London: Routledge. P.25

Radzinowicz, L. (2002). Adventures in criminology. London: Routledge.

Ruggiero, V., South, N., \& Taylor, I. (1998). The new European criminology. London: Routledge.

Sennett, R. (2011). El declive del hombre público. Barcelona: Anagrama.

Sutherland, E., \& Cressey, D. (1966). Principles of criminology. Philadelphia: Lippincott.

Walklate, S. (2007). Understanding criminology. Maidenhead: McGraw-Hill/Open University Press.

Wilhelm Heitmeyer. Control of violence. New York: Springer, 2011, p. 168.

Zaffaroni.E., (2011). La palabra de los muertos: Conferencias de criminología cautelar. Buenos Aires: Ediar, 2011

Zaffaroni, E. (2013). La emergencia del miedo. Buenos Aires, Argentina: Ediar.

Zaffaroni, E. (2005). En torno de la cuestión penal. Montevideo: B de F.

Zaffaroni, E., \& Rep. (2011). La cuestión criminal. C.A.B.A. [i. e. Ciudad Autónoma de Buenos Aires]: Planeta. 